\title{
Feridas invisíveis: Os impactos da mastectomia na autoimagem da mulher
}

\author{
Invisible wounds: The impacts of mastectomy on women's self-image \\ Heridas invisibles: Los impactos de la mastectomía em la autoimagen de las mujeres
}

Valéria Fernandes da Silva Lima ORCID: https://orcid.org/0000-0002-7516-4806

Universidade Estadual do Maranhão, Brasil E-mail: valeriafernandesxp@gmail.com Jéssica Maria Gomes Araújo ORCID: https://orcid.org/0000-0002-1341-8049 Universidade Regional do Cariri, Brasil E-mail: jessicamaria975@gmail.com

Sara Éllen Rodrigues de Lima

ORCID: https://orcid.org/0000-0001-6325-8568 Universidade Regional do Cariri, Brasil E-mail: sara.elima@outlook.com

Susiany Ferreira de Oliveira ORCID: https://orcid.org/0000-0003-1829-2534 Universidade Regional do Cariri, Brasil E-mail: susiany86@gmail.com

Luciano Santos da Silva Filho

ORCID: https://orcid.org/0000-0003-2325-3716 Escola de Saúde Pública do Ceará, Brasil E-mail: lucianofilhofisio@gmail.com

\begin{abstract}
Resumo
A realização da mastectomia na mulher traz à tona sentimentos e percepções negativas quanto a sua autoimagem, o que consequentemente afeta a autoestima e corrobora para problemas relacionados à sexualidade e relações sociais. $\mathrm{O}$ estudo tem como objetivo identificar mediante a literatura científica as repercussões na autoimagem das mulheres mastectomizadas. Trata-se de uma revisão integrativa da literatura com abordagem qualitativa, realizada na base de dados, LILACS e nas bibliotecas BVS e SCIELO. Utilizou-se os Descritores em Ciências da Saúde: Saúde da Mulher, Mastectomia e Autoimagem, que foram cruzados simultaneamente com o operador booleano AND. A seleção dos artigos utilizou como base os critérios de inclusão e exclusão, sendo selecionados uma amostra final de oito referências para compor o estudo. Após a retirada do seio, as mulheres apresentaram sentimentos de desespero, desorientação, chateação, inferioridade, medo da morte, constrangimento nas relações sexuais e perda da libido. A respeito da autoimagem destaca-se tristeza, pois entende-se que a retirada da mama afetará a feminilidade. Evidenciase a necessidade de elaboração de estratégias que visem a preparação das mulheres que serão submetidas a mastectomia, visando a minimização do sofrimento psíquico e a melhora de sua qualidade de vida.
\end{abstract}

Palavras-chave: Saúde da mulher; Mastectomia; Autoimagem.

\begin{abstract}
The performance of mastectomy in women brings up negative feelings and perceptions about their self-image, which consequently affects self-esteem and corroborates problems related to sexuality and social relationships. The study aims to identify through the scientific literature the repercussions on the self-image of mastectomized women. This is an integrative literature review with a qualitative approach, carried out in the database, LILACS and in the VHL and SCIELO libraries. The Health Sciences Descriptors: Women's Health, Mastectomy and Self-image were used, which were crossed simultaneously with the Boolean operator AND. The selection of articles used the inclusion and exclusion criteria as a basis, and a final sample of eight references was selected to compose the study. After withdrawing the breast, women showed feelings of despair, disorientation, upset, inferiority, fear of death, embarrassment in sexual relations and loss of libido. Regarding self-image, sadness stands out, as it is understood that breast removal will affect femininity. The need to develop strategies aimed at preparing women who will undergo mastectomy is evident, aiming at minimizing psychological distress and improving their quality of life.
\end{abstract}

Keywords: Women's health; Matectomy; Self image.

\section{Resumen}

La realización de mastectomía en mujeres hace surgir sentimientos y percepciones negativas sobre su autoimagen, lo que en consecuencia afecta la autoestima y corrobora problemas relacionados con la sexualidad y las relaciones sociales. El estúdio tiene como objetivo identificar las repercusiones en la autoimagen de las mujeres 
mastectomizadas a través de la literatura científica. MÉTODO: Se trata de una revisión bibliográfica integradora con enfoque cualitativo, realizada en la base de datos LILACS y en las bibliotecas BVS y SCIELO. Se utilizaron los Descriptores de Ciencias de la Salud: Salud de la Mujer, Mastectomía y Autoimagen, que se cruzaron simultáneamente con el operador booleano AND. La selección de artículos se basó en los criterios de inclusión y exclusión y se seleccionó una muestra final de ocho referencias para componer el estudio. Tras la retirada del pecho, las mujeres mostraron sentimientos de desesperación, desorientación, malestar, inferioridad, miedo a la muerte, vergüenza en las relaciones sexuales y pérdida de la libido. En cuanto a la autoimagen, destaca la tristeza, ya que se entiende que la extracción de los senos afectará la feminidad. Es evidente la necesidad de desarrollar estrategias encaminadas a preparar a las mujeres que serán sometidas a mastectomía, con el objetivo de minimizar el malestar psicológico y mejorar su calidad de vida.

Palabras clave: Salud de la mujer; Mastectomía; Auto imagen.

\section{Introduçãa}

O câncer de mama é uma patologia causada pela proliferação desordenada das células mamárias, propiciando a formação de um tumor. Mundialmente, esse tipo de câncer é o mais prevalente entre as mulheres, caracterizando-se como um importante problema de saúde pública, estima-se que 66.280 novos casos para cada ano do triênio 2020-2022 no Brasil (Brasil, 2019).

Nesse contexto a mastectomia é uma terapêutica que consiste na ablação da mama, podendo variar mediante a dimensão tumoral afetada, vale ressaltar que por se tratar de um procedimento invasivo, é capaz de desenvolver algumas complicações na saúde da mulher (Pereira, Gomes \& Oliveira, 2017). Além disso, a retirada da mama é um evento traumático com efeitos vigorosamente negativos, instigando emoções discrepantes que podem levá-las ao sofrimento psicossocial (Koçan \& Gursoy, 2016).

Posteriormente a mastectomia, a mulher passa por um período de vulnerabilidade emocional marcada por percepções negativas associadas à autoimagem e, por conseguinte na autoestima, refletindo em problemas na sua sexualidade, nas relações sociais e conjugais (Pereira, Gomes \& Oliveira, 2017).

Percebe-se que um dos principais impactos da mastectomia é na feminilidade da mulher, partindo da premissa de que a perda das mamas pode representar para elas a imagem de feias, incompletas, menos femininas e atraentes. Essas concepções prejudicam a autoestima e promovem inseguranças que comprometem sua sexualidade, pois os seios desempenham uma função importante na maternidade, sexualidade e vaidade (Koçan \& Gursoy, 2016; Lorenz, Lohmann \& Pissaia, 2019).

Em muitos casos, a mulher não possui preparo psicológico suficiente para se adaptar às mudanças ocorridas após a cirurgia (Rocha, Cruz, Vieira, Costa \& Lima, 2016). Comumente, as mulheres que se submeterão a uma mastectomia possuem diversos questionamentos acerca dos cuidados pós-cirúrgicos, se haverá necessidade de tratamento posteriormente, se o câncer será realmente findado após a cirurgia, dentre outros. E por vezes, a preocupação relacionada ao procedimento cirúrgico em demasia associada à ausência de informações fazem com que conflitos emocionais e pessoais se sobressaiam somente após a cirurgia.

Tendo-se isso em vista, uma mulher que possui os questionamentos esclarecidos acerca da cirurgia e do seu próprio corpo, paralelamente, detentora de uma autoestima elevada consegue enfrentar mais positivamente as mudanças que virão em decorrência da mastectomia. Assim, através do apoio, sobretudo, emocional e psicológico, as mulheres conseguem ressignificar em suas vidas, conceitos padronizados, como harmonização e erotização do corpo feminino, que não são perdidos após a cirurgia, e sim remodelados. Diante disso, este trabalho objetivou identificar mediante a literatura científica as repercussões geradas na autoimagem de mulheres submetidas a mastectomia. 


\section{Metodologia}

Trata-se de uma revisão integrativa da literatura com abordagem qualitativa. Para a elaboração do presente estudo, seguiu-se as seguintes etapas: identificação do tema e formulação da questão norteadora; delimitação dos critérios de inclusão e exclusão; seleção; análise dos estudos escolhidos; seleção das principais informações; interpretação dos resultados e apresentação da revisão integrativa (Mendes, Silveira \& Galvão, 2008).

Na primeira etapa formulou-se a questão norteadora com base no método PVO (População, Variável e Resultados). Resultando na pergunta norteadora: Quais os impactos observados na autoimagem de mulheres no pós-cirúrgico da mastectomia? Em seguida, foi definido os critérios de inclusão: artigos que versassem sobre os impactos observados na autoimagem de mulheres pós mastectomia; publicados nos idiomas português, inglês e espanhol; e disponíveis gratuitamente com texto completo. Ressalta-se que não foram encontrados artigos no idioma espanhol. Foram excluídos estudos que não atendiam a proposta da temática ou que não respondiam ao objetivo proposto; com download indisponível; teses, dissertações e outras revisões. Destaca-se que o recorte temporal foi de 10 anos para o maior alcance possível de publicações que pudessem responder à questão norteadora deste estudo.

A coleta de dados foi realizada na base de dados, Literatura Latino-Americana e do Caribe em Ciências da Saúde (LILACS) e nas bibliotecas, Biblioteca Virtual em Saúde (BVS) e na Scientific Electronic Library Online (SCIELO), utilizando o método de busca avançada. Adotou-se, para a busca, uma associação simultânea de três descritores: Saúde da Mulher, Mastectomia e Autoimagem, obtidos pelos Descritores em Ciências da Saúde (DeCS) e, para o cruzamento dos descritores, empregou-se o operador booleano AND.

Foram identificados 124 estudos inicialmente. Estes foram submetidos a um processo de análise de título e resumo, seguindo os critérios de inclusão e exclusão. Sendo realizada a seleção final dos estudos elegíveis para leitura da íntegra.

O processo de identificação dos artigos contou com a participação de dois pesquisadores, juntos padronizaram o processo de busca dos estudos, sendo executado de forma separada por cada autor. Ao término da seleção ocorreu a conferência dos resultados obtidos.

Destaca-se que foi utilizado o instrumento Preferred Reporting Items for Systematic Review and Meta-Analyses (PRISMA) para evidenciar o processo de identificação, seleção, elegibilidade e inclusão dos estudos, conforme representado no Quadro 1. 
Quadro 1. Processo de busca e seleção dos artigos.

\begin{tabular}{|c|c|c|c|c|}
\hline BASE/BIBLIOTECA DE DADOS & BVS & SCIELO & PUBMED & LILACS \\
\hline $\begin{array}{c}\text { Estudos identificados por meio da busca } \\
\text { bibliográfica nas bases de dados com o } \\
\text { uso dos descritores: Saúde da Mulher, } \\
\text { Mastectomia e Autoimagem. }\end{array}$ & 28 artigos & 1 artigo & 89 artigos & 6 artigos \\
\hline $\begin{array}{c}\text { Estudos rastreados: Artigo completo, } \\
\text { Idiomas Inglês e Português, recorte } \\
\text { temporal a partir de 2011. }\end{array}$ & 7 artigos & 1 artigo & 7 artigos & 1 artigos \\
\hline $\begin{array}{c}\text { 1 exclusão: } \\
\text { Excluídos a partir dos critérios de } \\
\text { inclusão (atender ao objetivo e ter } \\
\text { relação com a temática). }\end{array}$ & 3 artigos & 0 artigos & 5 artigos & 0 artigos \\
\hline $\begin{array}{c}\text { Estudos em texto avaliados para } \\
\text { elegibilidade: Leitura completa do } \\
\text { artigo. }\end{array}$ & 4 artigos & 1 artigo & 2 artigos & 1 artigo \\
\hline $\begin{array}{c}\text { Amostra final: Estudos incluídos na } \\
\text { síntese, os quais decorrem sobre o tema. }\end{array}$ & & 8 artigos & \\
\hline
\end{tabular}

Fonte: Autores (2021).

\section{Resultados e Discussão}

Quadro 2. Resultados encontrados nos artigos.

\begin{tabular}{|c|c|c|c|c|}
\hline $\begin{array}{c}\text { No DO } \\
\text { ARTIGO }\end{array}$ & TÍTULO & $\begin{array}{c}\text { AUTORES/ } \\
\text { ANO }\end{array}$ & OBJETIVO & SíNTESE DOS RESULTADOS \\
\hline A1 & $\begin{array}{c}\text { vivenciados pelas } \\
\text { mulheres } \\
\text { mastectomizadas }\end{array}$ & $\begin{array}{c}\text { Lima et al. } \\
2018\end{array}$ & $\begin{array}{c}\text { Sentimentos das } \\
\text { mulheres } \\
\text { mastectomizadas. }\end{array}$ & $\begin{array}{c}\text { Desvelar os } \\
\text { Identificou-se como sentimentos negativos frente } \\
\text { ao diagnóstico, desespero, tristeza, preocupação, } \\
\text { medo da morte e sentimento de inferioridade. A } \\
\text { respeito da autoimagem destaca-se tristeza, por } \\
\text { ser o símbolo da maternidade, sexualidade e } \\
\text { sensualidade, pois entende-se que a retirada da } \\
\text { mamafetará a feminilidade. Entretanto, destaca- } \\
\text { se o sentimento de esperança e alívio por parte de } \\
\text { algumas mulheres ao realizar a mastectomia. }\end{array}$ \\
\hline A2 & $\begin{array}{c}\text { Percepção das } \\
\text { mulheres } \\
\text { mastectomizadas } \\
\text { sobre a cirurgia } \\
\text { reconstrutiva da }\end{array}$ & $\begin{array}{c}\text { Silva et al. } \\
2017\end{array}$ & $\begin{array}{c}\text { percepção das } \\
\text { mulheres } \\
\text { mastectomizadas } \\
\text { sobre a cirurgia }\end{array}$ & $\begin{array}{c}\text { Analisar a } \\
\text { tristeza, desgosto, vergonha, insatisfação. Outra } \\
\text { questão levantada foi o uso de artifícios para } \\
\text { toalha, prótese, sutiã com enchimento e até }\end{array}$ \\
\hline
\end{tabular}




\begin{tabular}{|c|c|c|c|c|}
\hline & mama & & $\begin{array}{l}\text { reconstrutiva da } \\
\text { mama e os } \\
\text { sentimentos acerca } \\
\text { de sua condição. }\end{array}$ & $\begin{array}{c}\text { mesmo o CRM. Foram citadas também } \\
\text { limitações físicas e funcionais, como cansaço, } \\
\text { desconforto e restrições de movimento no braço } \\
\text { correspondente à mama extraída. }\end{array}$ \\
\hline $\mathbf{A 3}$ & $\begin{array}{c}\text { Mastectomia: as } \\
\text { cicatrizes na } \\
\text { sexualidade feminina }\end{array}$ & $\begin{array}{l}\text { Rocha et al. } \\
2016\end{array}$ & $\begin{array}{c}\text { Descrever os } \\
\text { reflexos da } \\
\text { mastectomia sobre a } \\
\text { sexualidade das } \\
\text { mulheres atendidas } \\
\text { no Programa Saúde } \\
\text { da Mulher. }\end{array}$ & $\begin{array}{l}\text { Identificou-se que após a retirada do seio, as } \\
\text { mulheres apresentaram sentimentos de desespero, } \\
\text { desorientação, chateação, inferioridade, medo da } \\
\text { morte, constrangimento nas relações sexuais e } \\
\text { perda da libido, uma vez que para elas a mama é } \\
\text { o órgão mais importante para a mulher. }\end{array}$ \\
\hline A4 & $\begin{array}{l}\text { Repercussão dos } \\
\text { efeitos da cirurgia } \\
\text { reconstrutora na vida } \\
\text { de mulheres com } \\
\text { neoplasias da mama. }\end{array}$ & $\begin{array}{c}\text { Inocenti et al. } \\
2016\end{array}$ & $\begin{array}{l}\text { Compreender a } \\
\text { repercussão dos } \\
\text { efeitos da cirurgia } \\
\text { reconstrutora na } \\
\text { vida de mulheres } \\
\text { com câncer de } \\
\text { mama. }\end{array}$ & $\begin{array}{l}\text { O estudo observou que a recuperação da mama } \\
\text { por meio do enxerto diminui os sentimentos } \\
\text { negativos que foram aflorados. Além disso, } \\
\text { devolve uma imagem que possibilita segurança, } \\
\text { reduzindo assim o trauma e melhorando a } \\
\text { qualidade de vida da mulher, assim como também } \\
\text { permite que elas se sintam à vontade para usar os } \\
\text { diferentes tipos de roupas. No entanto, algumas } \\
\text { participantes relataram ainda ter sentimentos de } \\
\text { insatisfação, pois não recuperaram a imagem } \\
\text { esperada. }\end{array}$ \\
\hline A5 & $\begin{array}{l}\text { Autoestima e } \\
\text { qualidade de vida de } \\
\text { mulheres submetidas } \\
\text { à cirurgia oncológica } \\
\text { de mama }\end{array}$ & $\begin{array}{l}\text { Gomes, S. N; } \\
\text { Soares, O. B. } \\
\quad \text { M; } \\
\text { Silva, R. S. } \\
\quad 2015\end{array}$ & $\begin{array}{c}\text { Analisar a } \\
\text { influência das } \\
\text { variáveis } \\
\text { sociodemográficas, } \\
\text { clínicas e } \\
\text { autoestima na } \\
\text { qualidade de vida } \\
\text { de mulheres } \\
\text { submetidas à } \\
\text { cirurgia oncológica } \\
\text { de mama. }\end{array}$ & $\begin{array}{l}\text { O estudo aponta que os aspectos relacionados à } \\
\text { idade, renda, escolaridade e o tempo de cirurgia, } \\
\text { influenciam nos fatores físico, psicológico, } \\
\text { ambiental e social dessas mulheres. Ressalta-se } \\
\text { que a adequada autoestima está fortemente } \\
\text { correlacionada às questões psicológicas e físicas, } \\
\text { corroborando assim para a importância do } \\
\text { sentimento positivo diante de todo o processo. }\end{array}$ \\
\hline A6 & $\begin{array}{c}\text { Impacto da } \\
\text { reconstrução } \\
\text { mamária na } \\
\text { qualidade de vida de } \\
\text { pacientes } \\
\text { mastectomizadas } \\
\text { atendidas no Serviço } \\
\text { de Cirurgia Plástica } \\
\text { do Hospital } \\
\text { Universitário Walter } \\
\text { Cantídio }\end{array}$ & $\begin{array}{c}\text { Paredes et al. } \\
2013\end{array}$ & $\begin{array}{l}\text { Verificar a } \\
\text { qualidade de vida } \\
\text { de pacientes } \\
\text { mastectomizadas e } \\
\text { submetidas a } \\
\text { reconstrução } \\
\text { mamária imediata } \\
\text { ou tardia, } \\
\text { abordando os } \\
\text { domínios físico, } \\
\text { psicológico e social. }\end{array}$ & $\begin{array}{l}\text { Identificou-se que a reconstrução da mama } \\
\text { possibilita à mulher mastectomizada uma maior } \\
\text { qualidade de vida, integridade, preservação da } \\
\text { sua autoimagem e diminuição de sentimentos } \\
\text { negativos, bem como um processo de reabilitação } \\
\text { menos traumático, ocasionando assim benefícios } \\
\text { físicos, psicológicos e sociais. Além disso, } \\
\text { observou-se que as mulheres submetidas a } \\
\text { reconstrução mamária tardia ficam mais } \\
\text { satisfeitas com o resultado, sendo justificado } \\
\text { devido ao fato de que tiveram maior tempo para } \\
\text { elaborar e encontrar novos significados para o } \\
\text { que vivenciaram. }\end{array}$ \\
\hline
\end{tabular}




\begin{tabular}{|c|c|c|c|c|}
\hline A7 & $\begin{array}{c}\text { Body Image of } \\
\text { Women with Breast } \\
\text { Cancer After } \\
\text { Mastectomy: A } \\
\text { Qualitative Research }\end{array}$ & $\begin{array}{l}\text { Koçan, S.; } \\
\text { Gursoy, A. } \\
2016\end{array}$ & $\begin{array}{c}\text { Obter uma } \\
\text { compreensão } \\
\text { holística e profunda } \\
\text { sobre como a } \\
\text { mastectomia afeta a } \\
\text { imagem corporal de } \\
\text { mulheres com } \\
\text { câncer de mama. }\end{array}$ & $\begin{array}{l}\text { Identificou-se que o seio significa feminilidade, } \\
\text { beleza e maternidade. O significado da } \\
\text { mastectomia é variável, as mulheres } \\
\text { demonstraram opiniões negativas sobre sua } \\
\text { aparência, bem como disseram sentir falta de uma } \\
\text { parte do corpo. Com relação à vestimenta, as } \\
\text { mulheres afirmaram preferir roupas que } \\
\text { escondessem a falta do seio, e algumas afirmaram } \\
\text { que as relações com os maridos já não eram como } \\
\text { antes. }\end{array}$ \\
\hline A8 & $\begin{array}{l}\text { Quality of Life of } \\
\text { Nepalese Women } \\
\text { Post Mastectomy }\end{array}$ & $\begin{array}{c}\text { Maharjan et al. } \\
2018\end{array}$ & $\begin{array}{c}\text { Determinar a } \\
\text { qualidade de vida } \\
\text { de mulheres } \\
\text { nepalesas após a } \\
\text { mastectomia. }\end{array}$ & $\begin{array}{l}\text { O estudo relata que as entrevistadas apresentaram } \\
\text { um bom índice de qualidade de vida, entretanto } \\
\text { obtiveram baixo desempenho com relação à } \\
\text { função sexual e ao prazer sexual. Já com relação } \\
\text { à mama, os efeitos colaterais apresentaram } \\
\text { associações com a idade, estado civil, ocupação, } \\
\text { educação, uso de prótese mamária e } \\
\text { comorbidades. Sendo a imagem corporal } \\
\text { fortemente associada com estes últimos aspectos. }\end{array}$ \\
\hline
\end{tabular}

Fonte: Autores (2021).

Após leitura e análise minuciosa, oito estudos foram eleitos para compor o presente trabalho. Esses apresentaram perspectivas abrangentes e análogas nas respectivas pesquisas encontradas sobre a temática em estudo, desde os títulos, objetivos e concepções metodológicas, apesar de um tanto discrepante quando comparado ao objetivo deste estudo, pois este restringe-se a responder sobre os impactos da mastectomia na autoimagem da mulher, mas é possível detectar nos resultados fragmentos que correspondem a temática. No entanto, no que concerne ao processo metodológico dos estudos, denota-se que a maioria das pesquisas, tratam de estudos descritivos com abordagens qualitativas (3), estudos transversais (2), transversal descritivo (1), exploratório-descritivo com abordagem qualitativa (1) e quanti qualitativo descritivo e exploratório (1). Em relação à localidade, 6 artigos foram publicados no Brasil e 1 nos EUA e Ásia respectivamente.

O câncer de mama é uma das patologias mais temidas pelas mulheres, em virtude da sua magnitude, quadro evolutivo impreterível e alterações estéticas promovidas pela mutilação, que causa significativas alterações na autoimagem. A terapêutica da doença traz consigo repercussões biopsicossociais provocadas pelas incertezas, medos, ansiedade e as modificações físicas (Rocha et al., 2016). Assim, o que pode parecer fácil e de tratamento imediato para o câncer de mama, passa a ser um pesadelo na vida das mulheres mastectomizadas, uma vez que os seios estão atrelados à autoestima da mulher, que transpassa muito além da aparência externa, é um ato de aceitação que ocorre de dentro para fora (Gomes \& Silva, 2013).

Dessa forma, a mastectomia é uma intervenção cirúrgica que baseia-se na extração da glândula mamária e músculos peitorais, com a finalidade de minimizar a possibilidade de ocorrência do câncer de mama, assim como assegurar uma melhora na expectativa e qualidade de vida das mulheres avaliadas com elevado risco (Lima et al., 2018). No entanto, o método debilita a forma como as mulheres visualizam sua feminilidade sendo afetada de forma inconversível (Silva, Ferreira, Ferro, Rocha \& Cavalcante, 2017). Ademais, a escolha pelo tratamento cirúrgico possui laboriosos impactos emocionais, psicológicos e funcionais para a vida, uma vez que a mama é considerada uma das partes mais femininas pelas mulheres, já que elas estão intrinsecamente ligadas à maternidade, sexualidade e feminilidade (Santos, Freitas, Andrade \& Silva2019).

De acordo com o estudo de Lima et al. (2018), a maioria das mulheres mastectomizadas possui uma faixa etária superior a 40 anos de idade. Resultado semelhante foi encontrado por Koçan \& Gursoy (2016), Gomes et al. (2015) \& 
Mahrjan et al. (2018) o qual é um fator que exerce influência sobre a qualidade de vida das mulheres submetidas ao tratamento cirúrgico, posto que mulheres mais jovens apresentam maiores dificuldades em adaptarem-se a sua nova realidade; as mulheres mais velhas, por sua vez, possuem mais apreço pela saúde e valorizam menos as mamas concomitantemente a sua feminilidade, autoimagem e sexualidade. No último caso, a relação com o parceiro restringe-se especialmente ao companheirismo, para essas o que realmente importa é a cura (Silva et al.,2017).

Em pesquisa realizada por Lima et al. (2018), a qual pretendia investigar os sentimentos de 25 mulheres submetidas a mastectomia, contatou-se em sua maioria sentimentos de tristeza, inferioridade, desespero, medo, preocupações com o futuro, juntamente com o receio eminente da morte e extração total das glândulas mamárias, tendo em vista que este prejudica a percepção de sua autoimagem. Sentimentos semelhantes foram evidenciados por (Rocha et al., 2016), em seu estudo com 14 mulheres compelidas ao procedimento, as quais relataram emoções de desespero, tristeza, chateação, horror e medo de não sobreviver. Posteriormente, ao procedimento cirúrgico foi observado sentimentos de dor, tristeza e sofrimento, ao mesmo passo que é identificado em algumas mulheres sensações de alívio, pois o procedimento auxilia na manutenção e revigoração para o estabelecimento da saúde (Lima et al., 2018).

Todavia, foi observado que uma pequena parcela afirmou tranquilidade ao receber e enfrentar o câncer de mama entende-se com isso, que estas - mulheres - objetivavam e mentalizavam a necessidade de serem fortes para enfrentar a doença e permanecerem firmes durante o árduo tratamento (Lima et al., 2018).

A tristeza em decorrência da ablação da mama é a emoção mais citada, visto que a excisão de uma parte tão importante do corpo carregado de significados subjetivos, fundamentais para a reafirmação da feminilidade, ocasiona em transformações quanto à percepção da mulher frente a sua imagem corporal, principalmente devido aos padrões de beleza introduzidos pela sociedade contemporânea, padrões que atribuem valores aos corpos femininos, especialmente aos seios que por vezes assumem papel de destaque, sendo vistos como símbolo de sensualidade e feminilidade (Lima et al., 2018).

Estudos apontam uma preocupação por parte das mulheres quanto à estética corporal após a realização da mastectomia. Evidenciou que a mastectomia provoca modificações na imagem corporal, exercendo influências negativas a autoestima e vaidade, interferindo na simbologia feminina a qual é atribuído importante acepção tanto para a mulher quanto para a sociedade que marginaliza os corpos que não se enquadram aos padrões (Silva et al., 2017; Rocha et al.,2016).

Paredes et al. (2013) \& Inocenti et al. (2016), identificaram em seus estudos que a reconstrução mamária possibilita às mulheres uma melhora na qualidade de vida, na sua integridade e preservação da autoimagem, minimizando os sentimentos negativos associados a ablação da mama e proporcionado a superação do evento traumático designado pela mutilação, permitindo assim o resgate da autoestima, a remodelação da autoimagem e restauração da sensualidade e feminilidade, resultando em benefícios a saúde biopsicossocial e consequentemente as relações afetivas. Em contrapartida, as entrevistadas do estudo de (Silva et al., 2017, em sua maioria, não mostraram interesse na reconstrução mamária, por medo do enfrentamento a uma nova cirurgia associada às incertezas da vida.

Comumente as mulheres sofrem com a autopercepção negativa da sua autoimagem, e expressam extrema preocupação com sua própria família e como outras pessoas percebem seus corpos após a mastectomia, por medo de rejeição, o que dificulta suas relações afetivas e sociais (Koçan \& Gursoy, 2016; Rocha et al.,2016). Portanto, a aceitação e ajuda do companheiro e da família, neste momento de fragilidade, é retratada como essencial para a descoberta e aceitação da nova imagem corporal (Inocente et al., 2016). De acordo com (Rocha et al.,2016), há relatos de rejeição dos parceiros após a cirurgia, intensificando sentimentos de abandono e insuficiência nas mulheres, estas sentem-se incompletas e por conseguinte bloqueiam desejos de se relacionar. 
Silva et al. (2017) ainda salienta que perante toda a extensão causada na vida das mulheres, resultante do diagnóstico e do tratamento radical, foram notadas nas falas, expressões e ações que a fé em Deus e a contribuição da família e dos amigos mostraram-se fortes aliadas no enfrentamento da doença, haja vista, que o conforto, confiança, suporte, consolo e fortaleza contribuem para que o processo de aceitação da doença e o encorajamento e perseverança do tratamento não sejam vistos como algo impossível.

\section{Considerações Finais}

Diante de todo o processo de descoberta, tratamento e cirurgia do câncer de mama, a mulher vivencia em sua intrínseca singularidade experiências distintas, não sendo possível generalizar com objetividade seus sentimentos e reações a mastectomia, mas na maioria dos casos expressam sentimentos negativos como tristeza, desespero, medo e incertezas com relação ao futuro. Entretanto, após a mastectomia os sentimentos identificados foram dor, tristeza e sofrimento, mas também alívio em saber que a doença foi retirada do seu corpo. Quanto à sexualidade, identificou-se sentimentos de vergonha, diminuição da vontade da prática sexual e perda da sensibilidade na região da mama operada.

Neste contexto, observa-se que a extração da glândula mamária deixa as mulheres mais fragilizadas, uma vez que seus seios são órgãos que exercem papel de grande representatividade feminina, símbolos que representam a personificação da sensualidade, sexualidade, maternidade e estética corporal, e muitas são as emoções e percepções negativas acerca de sua autoimagem em decorrência deste evento traumático, prejudicando assim a autoestima e consequentemente a qualidade de vida e o bem estar físico e social. Portanto, é imprescindível a compreensão e apoio do companheiro, familiares e amigos para a superação dos conflitos internos provocados pela extração da mama.

Este estudo apresentou limitações quanto às informações acerca dessa temática, uma vez que é notória a escassez de estudos relacionados à percepção da autoimagem feminina mediante a mastectomia. Nesse sentido, é importante salientar que pesquisas nesse segmento se fazem necessárias tanto para subsidiar a delimitação de estratégias que visem a preparação das mulheres com câncer que serão submetidas a mastectomia, como para o acompanhamento posterior a cirurgia, buscando uma melhora na qualidade de vida e minimização do sofrimento psíquico dessas mulheres em virtude de sua autoimagem prejudicada.

\section{Referências}

Brasil. Instituto Nacional de Câncer. Coordenação de Prevenção e Vigilância. Estimativa 2020: incidência de câncer no Brasil. INCA; 2019.

Gomes, N. S., Soares, M. B. O., \& Silva, S. R. (2015). Self-Esteem and Quality of Life In Women Undergoing Breast Cancer Surgery. REME: Revista Mineira de Enfermagem, 19 (2), 120-132.

Gomes, N. S., \& Silva, S. R. (2013). Avaliação da autoestima de mulheres submetidas à cirurgia oncológica mamária. Texto e Contexto Enfermagem, 22 (2), $509-516$.

Inocenti, A., Santos, M. A., Loyola, E. A. C., Magalhães, P. A. P., \& Panobianco, M. S. (2016). Repercussão dos efeitos da cirurgia reconstrutora na vida de mulheres com neoplasias da mama. Texto e Contexto Enfermagem, 25 (2), 1-9.

Koçan, S., \& Gürsoy, A. (2016). Body image of women with breast cancer after mastectomy: a qualitative research. The journal of breast health, 12 (4), 145.

Lima. M. M. G., Leite. K. N. S., Santos, M. L. L., César, E. S. R., Souza, T. A., Nascimento, B. B., Barboza, J. P., \& Dantas, T. M. (2018). Sentimentos Vivenciados pelas mulheres mastectomizadas. Rev. Enferm. UFPE online, 12 (5), 1216-1224.

Lorenz, A. S., Lohmann, P. M., \& Pissaia, L. F. (2019). Impactos da mastectomia em mulheres diagnosticadas com câncer de mama em relação à autoimagem. Research, Society and Development, 8 (7), e8871099-8871099.

Maharjan, M., Thapa, N., Adhikari, R. D., Petrini, M. A., \& Amatya, K. S. (2018). Quality of life of Nepalese women post mastectomy. Asian Pacific Journal of Cancer Prevention: APJCP, 19 (4), 1005-1012.

Mendes, K. D. S., Silveira, R. C. C. P., \& Galvão, C. M. (2008). Revisão integrativa: método de pesquisa para a incorporação de evidências na saúde e na enfermagem. Texto \& Contexto - Enfermagem, 17 (4), 758-764. 
Research, Society and Development, v. 10, n. 4, e5810413800, 2021

(CC BY 4.0) | ISSN 2525-3409 | DOI: http://dx.doi.org/10.33448/rsd-v10i4.13800

Paredes, C. G., Pessoa, S. G. P., Peixoto, D. T. T., Amorim, D. N., Araújo, J. S., \& Barreto, P. R. A. (2013). Impacto da rec onstrução mamária na qualidade de vida de pacientes mastectomizadas atendidas no Serviço de Cirurgia Plástica do Hospital Universitário Walter Cantídio. Rev. Bras. Cir. Plást, 28 (1), 100-104.

Pereira, G. B., Gomes, A. M. S. M., \& Oliveira, R. R (2017). Impacto do tratamento do câncer de mama na autoimagem e nos relacionamentos afetivos de mulheres mastectomizadas. Life Style, 4 (1), 99-119.

Rocha, J. F. D., Cruz, P. K. R., Vieira, M. P., Costa, F. M., \& Lima, C. A. (2016). Mastectomia: As Cicatrizes Na Sexualidade Feminina. Rev. Enferm. UFPE online, $10(5), 4255-63$.

Santos, M. S., Freitas, D. F. L., Andrade, J. S. O., \& Silva, F. M. L. (2019). Implicações da mastectomia na autoestima da mulher. Revista Eletrônica Acervo Saúde, (29), e1124-1124.

Silva, J. B., Ferreira. C. B., Ferro, J. S., Rocha, L. O. M., \& Cavalcante, K. D. (2017) Percepção das mulheres mastectomizadas sobre a cirurgia reconstrutiva da mama. Rev. enferm. UFPE on line, 2056-2066. 